\title{
The physical properties of lipid monolayers and bilayers containing calixarenes sensitive to cytochrome $c^{*}$
}

\author{
Pavol Vitovic, Veronika Subjakova and Tibor Hianik \\ Department of Nuclear Physics and Biophysics, Faculty of Mathematics, Physics and Informatics, Comenius University, \\ Bratislava, Slovak Republic
}

\begin{abstract}
We studied physical properties of the monolayers and bilayer lipid membranes (BLM) formed by calix[6]arene carboxylic acid derivative (CX) and its mixtures with diphytanoylphosphatidylcholine (DPhPC) by means of measurement surface pressure, surface dipole potential and electrostriction. CX forms stable monolayers at an air-water interface and complexes in mixed monolayers contained DPhPC. Calixarenes increase the elastic moduli of lipid monolayers as well as BLM. Cytochrome c (cyt c) specifically binds to CX by incorporation of the amino groups of lysine residues at the protein surface. This binding affected the physical properties of CX monolayers depending on their initial surface pressure. Addition of cyt $\mathrm{c}$ into the water subphase induced increase of surface pressure of CX monolayers at relatively low initial pressure $(15 \mathrm{mN} / \mathrm{m})$ when monolayer was in liquid expanded state (LE). This may be due to interaction of positively charged cyt $\mathrm{c}$ with negatively charged carboxylic groups of CX and also by its penetration into the air-water interface. However, much subtle changes were observed for higher initial surface pressure (20 and 35 $\mathrm{mN} / \mathrm{m}$ ) when monolayer is in liquid condensed (LC) and solid (S) state, respectively. Lysine induced substantially lower changes in surface pressure in comparison with that of cyt $\mathrm{c}$.
\end{abstract}

Key words: Calixarene - Cytochrome c- Monolayers - Bilayer lipid membranes - Mechanical properties

Abbreviations: BLM, bilayer lipid membranes; cyt c, cytochrome c; CX, calix[6] arene carboxylic acid derivative; DPhPC, diphytanoylphosphatidylcholine; $\pi-\mathrm{A}$, surface pressure-area isotherm.

\section{Introduction}

Lipid monolayers and bilayer lipid membranes (BLM) are convenient models of biomembranes (Petty 1996; Hianik 2008). In recent years the interest to these structures has raised substantially due to their applications in nanotechnology, especially for development of chemical sensors and biosensors. Lipid bilayers are ordered but still sufficiently soft liquid-crystalline structures providing an optimal environ-

Correspondence to: Tibor Hianik, Faculty of Mathematics, Physics and Informatics, Comenius University, Mlynska dolina F1, 84248 Bratislava, Slovak Republic

E-mail: tibor.hianik@fmph.uniba.sk

* This article was presented at the $5^{\text {th }}$ Slovak Biophysical Symposium, organised by The Slovak Biophysical Society in Bratislava, March 19-21, 2012 ment for proper functioning of biopolymers and receptors (Nikolelis et al. 2010). Recent trends in sensor development are connected with incorporation of calixarenes into the lipid films as these devices could selectively determine various compounds, for example catecholamines (Nikolelis and Theoharis 2003). The calixarenes incorporated into the lipid membranes and monolayers can also serve as a model for the study of the mechanisms of ligand-receptor interactions (Zadmard et al. 2004).

Calix $[n]$ arenes are macrocyclic aromatic molecules originating from the synthesis of phenols and aldehydes, where [n] refers to the number of the phenol aromatic cycles in the molecule. The phenol subunits are bridged via methyl groups and thus provide the characteristic vase-like shape of calixarenes (Ludwig 2000; Ludwig and Dzung 2002). Due to the presence of the hydrophobic cavity formed by the phenol units, calixarenes have been successfully employed 
for detection of a wide range of compounds including nucleosides (Guo et al. 2004; Ludwig 2005; Cacciapaglia et al. 2006), amino acids (Hassen et al. 2007; Sugden et al. 2008), or proteins (Oh et al. 2005; Oshima et al. 2010). Moreover, modification of the side groups allows one to prepare tailormade calixarenes with high affinity towards the specific target molecules (Baldini et al. 2007; Sliwa and Deska 2011).

Monolayers formed by calix[n] arenes have been studied either on the water subphase (Omar et al. 1997; van der Heyden et al. 2002; Vollhardt et al. 2003; Lonetti et al. 2004), or on the subphases containing various cations (Dei et al. 1995; Capuzzi et al. 2000; Ye et al. 2001; Houel et al. 2002; Turshatov et al. 2004). In addition, calix [4] arenes monolayers have been shown to detect monomeric nucleosides in the subphase ( $\mathrm{Li}$ et al. 2001; Liu et al. 2002; Guo et al. 2004). The tetraanionic calixarene tetraphosphonate 1 incorporated into the stearic acid monolayer has benn shown as effective receptor for basic proteins such as cytochrome c (cyt c) (Zadmard et al. 2004). In our previous work we studied the physical properties of monolayers formed by calix[4] resorcinarene and in mixtures with dipalmitoylphosphatidylcholine (DPPC) in various molar ratios at the presence of dopamine in water subphase. We showed that both calix[4]resorcinarene, as well as its mixtures with DPPC, form stable monolayers. The presence of dopamine resulted in increase of the mean molecular area and in decrease of the compressibility modulus of the monolayers (Vitovic et al. 2006). A contactless method of recording Maxwell displacement current (MDC) enabled us to monitor changes in the charge state of the monolayer composed of the calix[4]resorcinarene-DPPC. We showed that the changes of the MDC started already in a gaseous state of the monolayer. Based on MDC we determined normal component of dipole moment of the calix[4]resorcinarene and its complex with dopamine, which reached maximal value of $1.04 \mathrm{D}$ at the region of the phase transition from liquid to solid state of the monolayer (Weis et al. 2007). Furthermore, BLM (Jin 2007) and liposomes (Sidorov et al. 2002; Izzo et al. 2008) were modified by calixarenes that form ionic channels for $\mathrm{Cl}^{-}$. The calixarene specific to cyt $c$ has also been reported (Oshima et al. 2005). This calix[6] arene carboxylic acid derivative (CX) is composed of 6 phenol rings and its cavity selectively bind amino group. Cyt c strongly binds to this calixarene due to 19 positively charged lysine residues terminated by amino groups at the cyt c surface. Most recently we demonstrated that CX can be incorporated into the supported lipid films (sBLM) (Mohsin et al. 2011) and into the large unilamellar vesicles (LUV) composed of dimyristoylphosphatidylcholine (DMPC) (Garaiova et al. 2012). Using impedance spectroscopy we have shown, that cyt c specifically interacts with sBLM modified by CX. At the same time with increased concentration of CX in LUV their diameter increased and zeta potential became more negative due to increased concentration of negatively charged carboxylic group of CX. At presence of cyt c, certain neutralization of negative surface charge took place. Thus it is likely that CX affects the physical properties of the lipid bilayer. We suspect that due to rather rigid structure of CX especially mechanical properties of both lipid monolayers and bilayers should be affected. In addition it would be rather interesting to compare effect of CX on monolayers and bilayers of identical composition. We have shown earlier, that increased concentration of cyt $c$ in a water subphase resulted in a shift of $\pi$-A isotherms formed by $\mathrm{CX}$ toward higher molecular area and determined equilibrium constant of dissociation $\mathrm{K}_{\mathrm{D}}=26.0 \pm 5.4 \mathrm{nM}$, which is evidence of good affinity of cyt $\mathrm{c}$ to the CX. The reason of high interest for detection cyt $\mathrm{c}$ as well as for analysis of its interaction with artificial receptors, such are calixarenes is due to an important biological role of this protein. Cyt $\mathrm{c}$ is small hemoprotein (molecular weight $12.4 \mathrm{kDa}$ ) located in the intermembrane space of mitochondria and plays a dual role in living system. It participates in electron transport and is responsible for the activation of the apoptotic pathway through releasing from mitochondria into the cytosol (Schug and Gottlieb 2009). The detection of endogenous concentration of cyt $\mathrm{c}$ is of high importance for diagnosis of possible pathological processes in the organism (Teuntje et al. 2010).

In this article we present the results of the studies of the physical properties of lipid monolayers and BLM composed of DPhPC with various molar ratio of the CX. DPhPC contain branched hydrocarbon chains and are important component of the membranes of halobacteria (Kates et al. 1965; Barcena-Uribarri et al. 2010). We showed that CX forms stable monolayers at an air-water interface, as well as stable mixed DPhPC/calixarene monolayers. CX also interacts favorably with lipids forming lipid-calixarene complexes. Stable BLM are formed also at presence of $\mathrm{CX}$ but only up to molar ratio $\mathrm{DPhPC} / \mathrm{CX}=50: 1$. The interfacial elastic modulus of area compressibility, $\mathrm{C}_{\mathrm{S}}{ }^{-1}$, of monolayers and Young modulus of elasticity of BLM, $\mathrm{E}_{\perp}$ increased with the increasing concentration of the CX. We also studied the interaction of cyt $\mathrm{c}$ with monolayers composed of CX, and showed that this protein induced increase of surface pressure depending on an initial surface pressure of the monolayer.

\section{Materials and Methods}

\section{Chemicals, preparation of BLM}

CX was gift of Dr. T. Oshima and has been synthesized as described in paper by Oshima et al. (2005), where also the structure of this molecule is presented. DPhPC was supplied by Avanti Polar Lipids Inc. (USA). Cyt c from bovine heart and lysine were supplied by Sigma-Aldrich (USA). Other 
chemicals were of p.a. grade and were used without further purification. The monolayers were prepared by spreading of a small amount $(20-23 \mu \mathrm{l})$ of the chloroform solution of calixarenes or their mixtures with DPhPC (concentration $1 \mathrm{mg} / \mathrm{ml}$ ) using the microsyringe (Hamilton, USA) on the water subphase of the Langmuir trough. The deionised water was prepared using ELIX 5 water purified system (Millipore, El Paso, USA). The DPhPC/calixarene mixtures were prepared at the molar ratios of 50:1, 30:1, 20:1 and 10:1. Each monolayer was allowed to equilibrate for $15 \mathrm{~min}$. This time was sufficient for solvent evaporation and monolayer stabilization.

BLMs were prepared from a mixture of DPhPC with cholesterol (Fluka, Switzerland) $(4: 1 \mathrm{w} / \mathrm{w})$ dissolved in $n$-heptane (Fluka) at a concentration of $20 \mathrm{mg} / \mathrm{ml}$ and contained calixarenes at the same molar ratio with DPhPC. Cholesterol was used for providing better stability of BLM. BLMs were formed according to Mueller et al. (1962) on a circular aperture of a $0.7 \mathrm{~mm}$ diameter in a Teflon partition dividing the Teflon cup into two identical compartments with a volume of $3.5 \mathrm{ml}$ each. Briefly, after accurate cleaning of the cell with detergent, washing with distilled water and drying at $80^{\circ} \mathrm{C}$, the aperture was pre-treated with a small amount of lipid solution (approx. $10 \mu \mathrm{l}$ ). After $n$-heptane evaporation during approx. $10 \mathrm{~min}$. and formation of a thin lipid layer at the aperture, the cell was filled with a buffer $(0.1 \mathrm{M} \mathrm{KCl}+50 \mathrm{mM}$ HEPES, pH 5.5). The membrane was formed by means of the Pasteur pipette soaked by a small amount of lipid solution. The pipette was placed at the bottom part of the aperture, where the bubble has been developed. The bubble first covered the aperture and then arose toward the water surface. The small amount of lipid film that remained attached to the aperture was sufficient for the formation of BLM. The film was first rather thick (several hundreds of $\mathrm{nm}$ ) as indicated by the appearance of colored Newton circles. However, as soon as the self-assembling thinning process started (almost immediately after appearance of the Newton circles), the black - bilayer regions appeared, rapidly expanding over the aperture. The formation of the membrane was observed optically by means of a stereomicroscope and electrically by measuring the electrical capacitance.

\section{Surface pressure-area $(\pi-A)$ isotherms and cyt c titration experiments}

$\pi$-A isotherms were measured using computer-controlled Langmuir trough 311D (NIMA Technology, Coventry, UK) made of Teflon (volume $\sim 200 \mathrm{ml}$, the whole surface area was $300 \mathrm{~cm}^{2}$ ) and Teflon moveable barrier. The through was equipped with Wilhelmy plate pressure sensor NIMA PS4. All monolayers were compressed at the constant speed $6 \mathrm{~cm}^{2} / \mathrm{min}$. The temperature of the subphase was maintained constant by thermostat Lauda RE206 (Köningshofen, Germany).

Titration experiments with cyt $\mathrm{c}$ or with lysine were conducted on the subphase buffered by PBS. First, small magnetic stirrer was placed on the bottom of the Teflone dish. Then, $7 \mathrm{ml}$ of PBS were added, and Wilhelmy plate was immersed into the subphase. Target surface pressure was obtained by spreading the appropriate volume of CX solution in chloroform (stock concentration $0.2 \mathrm{mg} / \mathrm{ml}$ ) on the surface of water subphase using the microsyringe until the target surface pressure $(5,15,20$ or $35 \mathrm{mN} / \mathrm{m})$ was reached and stabilized. Cyt c (stock solution $10 \mu \mathrm{M}$ in a PBS) or lysine (stock solution $100 \mu \mathrm{M}$ in a PBS) was titrated to the subphase via the small hole on the side of Teflone dish using the microsyringe. After the each titration step, system was allowed to equilibrate for 10-15 minutes until the surface pressure reached was stabilized. All the measurements were performed at $\mathrm{T}=24^{\circ} \mathrm{C}$.

\section{Surface dipole potential}

The surface dipole potential, $\Delta \Psi$, of the monolayer is a difference between the potential of a monolayer and that of pure subphase. The measurement of surface dipole potential allows one to analyze changes in the orientation of the molecular dipoles in the monolayer during compression. Presence of the monolayer between electrodes causes changes of the potential based on the Helmholtz equation:

$$
\Delta \Psi=\mu_{\mathrm{n}} /\left(\mathrm{A} . \varepsilon_{\mathrm{r}} \varepsilon_{\mathrm{o}}\right)
$$

where $\varepsilon_{\mathrm{r}}$ and $\varepsilon_{\mathrm{o}}$ are the relative dielectric constant and the permittivity of vacuum, respectively, $\mu_{n}$ is the normal component of the dipole moment of the molecule, and $\mathrm{A}$ is the molecular area. The surface dipole potential was measured by means of vibrating plate method (Brockman 1994) using high sensitive electrostatic voltmeter 320C and electrode 3250 (Kelvin probe) (TREK Inc., USA) mounted on special adjustable platform (NIMA). The probe was suspended in the air approx. $2 \mathrm{~mm}$ above the surface. The surface dipole potential was measured in respect to the pure subphase. The potential of later was taken as zero. The surface dipole potential was measured simultaneously with the surface pressure using NIMA interface and software.

The accuracy of measurement of the surface pressure and dipole potential was $0.1 \mathrm{mN} / \mathrm{m}$ and $1 \mathrm{mV}$, respectively.

\section{Measurement of $E_{\perp}$ by electrostriction method}

The electrostriction method enabled simultaneous measurement of the elasticity modulus in the direction perpendicular to the membrane plane $\mathrm{E}_{\perp}$, the coefficient of dynamic viscos- 
ity $\eta$, and the electrical capacitance $C$, of the bilayer. Briefly, an alternating voltage of amplitude $50 \mathrm{mV}$ was applied to the BLM through the reference $\mathrm{Ag} / \mathrm{AgCl}$ electrodes. Due to the non-linear dependence of capacitance on the voltage $\mathrm{V}(\mathrm{C}=$ $\mathrm{C}_{0}\left(1+\alpha \mathrm{V}^{2}\right)$, where $\mathrm{C}_{0}$ is the capacitance at $\mathrm{V}=0$ and $\alpha$ is the electrostriction coefficient), third current harmonic with frequency $3 \mathrm{f}$ and amplitude $\mathrm{A}_{3}$ is generated in addition to the basic first current harmonic, $A_{1}$ (frequency $\mathrm{f}=1 \mathrm{kHz}$ ). The measurements of these amplitudes allowed us to determine the elasticity modulus:

$$
\mathrm{E}_{\perp}=3 \mathrm{C}_{\mathrm{m}} \mathrm{V}_{0}^{2} /\left(4 \mathrm{hA}_{3}\right)
$$

where $C_{m}$ is the specific capacitance of the membrane $\left(C_{m}=\right.$ $\mathrm{C} / \mathrm{A}, \mathrm{A}$ is the membrane area), $\mathrm{V}_{0}$ is amplitude of $\mathrm{AC}$ voltage and $h$ is thickness of hydrophobic part of the membrane. The viscous properties of the BLM are characterized by coefficient of dynamic viscosity, $\eta$. This coefficient can be determined using following equation:

$$
\eta=E_{\perp} /(2 \pi f \sin \varphi)
$$

where $\varphi$ is the phase shift between third harmonic generated by BLM and input AC current. The membrane capacitance is determined by:

$$
\mathrm{C}=\mathrm{A}_{1} / 2 \pi \mathrm{fV} \mathrm{V}_{0}
$$

(see Hianik and Passechnik 1995, for details of the method and experimental set up).

All measurements on BLM were performed at $24^{\circ} \mathrm{C}$ and repeated at least six times to ensure the reproducibility of the results.

A

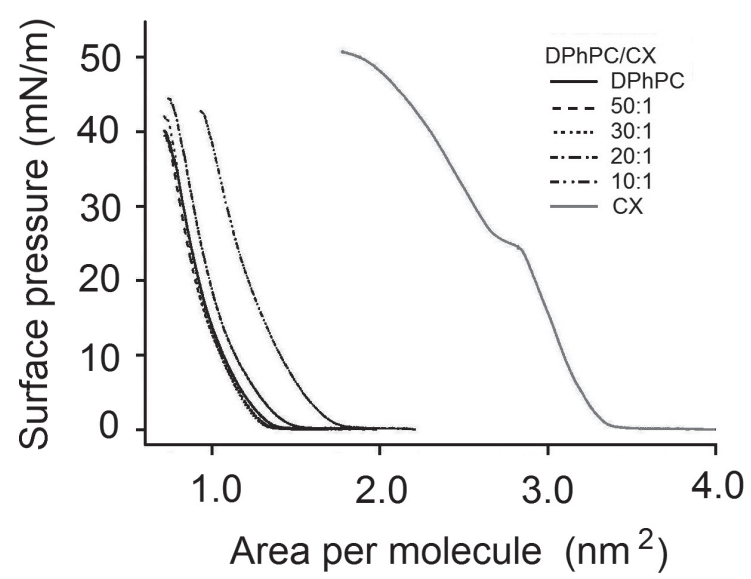

\section{Results and Discussion}

Pressure-area isotherms and surface dipole potential of the monolayers composed of CX and their mixtures with phospholipids

In the first series of experiments we studied the properties of monolayers composed of pure phospholipids, calixarenes and phospholipid-calixarene mixtures. So far, mostly DPPC has been used for studying of the properties of lipid monolayers at various modifications (Maget-Dana 1999). We used DPhPC in particularly due to the fact that this lipid has been shown to form stable BLM (see below). Certain similarity of DPhPC and DPPC consists in the same phosphocholine group and in the same number of carbons (16) in each of two hydrophobic chains. However, the structure of DPhPC is more robust due to 4 methyl residues at each hydrocarbon chains. Important peculiarity of branched hydrocarbon chains is that DPhPC does not exhibit a detectable gel to liquid crystalline phase transition in a wide temperature range $\left(-120\right.$ to $\left.+120^{\circ} \mathrm{C}\right)$ and has higher specific volume $(1.013 \mathrm{ml} / \mathrm{g})$ in comparison with that of DPPC $(0.950 \mathrm{ml} / \mathrm{g})$ (see Lindsey et al. 1979). DPhPC, similarly to other glycerophospholipids, forms stable monolayers at an air-water interface (Fig. 1). It is seen from Fig. 1A that under monolayer compression of pure DPhPC, the pressure starting to rise at $1.37 \mathrm{~nm}^{2} /$ molecule, which indicates transition from gaseous (G) to the liquid-expanded phase (LE). There is not clearly expressed transition from LE phase to liquid-condensed phase (LC), but the collapse of the monolayer took place at $\sim 40 \mathrm{mN} / \mathrm{m}$. However, there is an ongoing dispute on the presence of LC phase in DPhPC monolayers. Some authors did not report its existence (Lindsey et al. 1979), while oth-

B

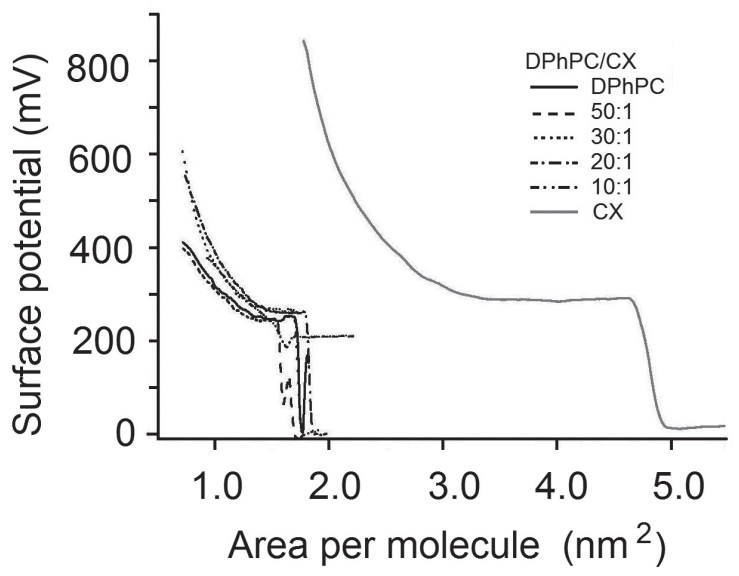

Figure 1. The plot of surface pressure (A) and surface dipole potential (B) as a function of mean molecular area for mixed monolayers composed of DPhPC and CX of different molar ratios (see the legend). 
ers observed this transition (Hsieh et al. 1997). The mean area per molecule of DPhPC in the LE phase is $1.04 \pm 0.02$ $\mathrm{nm}^{2}$, which agrees well with already published data (Cseh and Benz 1999; Guntupalli et al. 2011) (Table 1). In contrast to phospholipids without branched hydrocarbon chains, DPhPC did not reveal sharp pressure drop at the critical (collapse) pressure (Discher et al. 1999; Deshmukh et al. 2010). However, at the collapse region the elasticity modulus decreases rapidly similarly to other phospholipids, which is evidence of the monolayers disintegration.

Simultaneously with $\pi-\mathrm{A}$ isotherms we also measured surface dipole potential of the monolayers. This value is similar to both DPhPC and DPPC monolayers. At the beginning of the film compression the potential is $\sim 220 \mathrm{mV}$, and increases stepwisely to $\sim 450 \mathrm{mV}$ at the critical pressure (Fig. 1B). This behavior agrees well with those reported for DPPC monolayers (Shapovalov 1998). As it follows from $\pi$-A isotherms, the bulkier DPhPC molecules cause significant increase of mean molecular area in comparison to DPPC. However, the methyl residues have minor contribution into the surface dipole potential in comparison with amphiphilic group of the phospholipid. The fluctuations of the surface dipole potential at the beginning of compression observed for DPhPC, and also for mixed monolayers, are of mechanical nature possibly due to the barrier moving initialization. However, after the initial mechanical shock, and short fluctuations the potential got stabilized at the value $\sim 250 \mathrm{mV}$, and then gradually increased until the value of $400 \mathrm{mV}$ (for pure lipid monolayer) at the collapse pressure was reached.

CX forms stable monolayers at an air-water interface (Fig. $1 \mathrm{~A})$. The $\pi$-A isotherms are, however, different from previously reported calix [4] resorcinarene (Vitovic et al. 2006). In later case a sharp transition from gaseous to the solid phase took place already at $2.0 \mathrm{~nm}^{2}$ per molecule. The collapse of the monolayer started at $\sim 47 \mathrm{mN} / \mathrm{m}$. The transition of the monolayer composed of CX to the solid phase occurred at higher molecular areas, at $3.3 \mathrm{~nm}^{2}$ per molecule. New peculiarity of CX monolayers consists in the appearance of additional transition at surface pressure $\sim 25 \mathrm{mN} / \mathrm{m}$. The mean molecular area of CX is presented in Table 1. Relatively large molecular area of CX in comparison for example with four rings containing calix[4]resorcinarene (Vitovic et al. 2006) agrees well with the higher number of phenol rings.

The plot of surface dipole potentials of the CX monolayer vs. area per molecule is presented on Fig. 1B. It can be seen that the abrupt lift of the potential occurred already in gaseous phase and remained constant at the transition to the solid phase. Then, the potential had risen monotonously.

We also studied the properties of mixed DPhPC/calixarene monolayers. The corresponding $\pi$-A isotherms are presented on Fig. 1A. The mean molecular areas are listed in Table 1. As it can be seen from Fig. $1 \mathrm{~A}$ and Table 1, for mixed
Table 1. The properties of pure and mixed monolayers composed of DPhPC and CX in a various molar ratios

\begin{tabular}{ccc}
\hline $\begin{array}{c}\text { Monolayer composition } \\
\text { DPhPC }: \text { CX } \\
(\mathrm{mol} / \mathrm{mol})\end{array}$ & $\begin{array}{c}\text { Area per } \\
\text { molecule } \\
\left(\mathrm{nm}^{2}\right)\end{array}$ & $\begin{array}{c}\text { Modulus of area } \\
\text { compressibility } \\
(\mathrm{mN} / \mathrm{m})\end{array}$ \\
\hline $1: 0$ & $1.04 \pm 0.02$ & $104.5 \pm 2.0$ \\
$50: 1$ & $1.03 \pm 0.02$ & $101.1 \pm 1.9$ \\
$30: 1$ & $1.04 \pm 0.02$ & $116.8 \pm 2.0$ \\
$20: 1$ & $1.12 \pm 0.02$ & $108.2 \pm 2.0$ \\
$10: 1$ & $1.42 \pm 0.03$ & $104.0 \pm 1.8$ \\
$0: 1$ & $3.32 \pm 0.04$ & $195.3 \pm 2.1$ \\
\hline
\end{tabular}

Results represent mean \pm SD obtained from at least four experiments in each series.

monolayers containing CX in molar ratio DPhPC/CX 50:1 and 30:1 we obtained the mean molecular areas even lower in comparison to pure DPhPC. This effect is observed when interactions between phospholipid and calixarenes result in the energetically favorable formation of the complexes that evoke better ordering of the monolayers (Slottrup et al. 2005). At the same time the content of CX in a monolayer at above-mentioned molar ratios is relatively small. The mean molecular areas for molar ratios 50:1 and 30:1 differ not more than by $0.5 \%$.

A more detailed insight into the miscibility of monolayer components can be obtained by calculating the excess area as compared to the ideal mixing. For a given surface pressure the excess area, $A_{12}$, is related to the pure components as follows (Maget-Dana 1999):

$$
\mathrm{A}_{12}=\mathrm{XA}_{1}+(1-\mathrm{X}) \mathrm{A}_{2}
$$

where $A_{1}$ and $A_{2}$ are the molecular areas of calixarene and DPhPC, respectively. $A_{12}$ is the average area per molecule of the mixed monolayer and $\mathrm{X}$ is the molar fraction of calixarene. In the case of ideal miscibility, the plot of $\mathrm{A}_{12} v s$. $\mathrm{X}$ should be straight line connecting the values of mean molecular areas of pure components. Negative deviation from ideal miscibility indicates interaction between molecules, e.g. formation of DPhPC/CX complexes, while positive deviation can be related to formation of aggregates of pure components (Maget-Dana 1999). In order to analyze the influence of CX on monolayers we constructed the plots of the mean molecular area as a function of molar fraction of calixarenes at three different surface pressures, at which the monolayers are considered to be at structurally different phases: 5, 20 and $30 \mathrm{mN} / \mathrm{m}$. These plots are presented on Fig. 2. It can be seen that the deviation of area per molecule from that corresponding to ideal miscibility is rather small. Therefore, based on the data presented in Table 1 we constructed the plot of area per molecule versus relative 


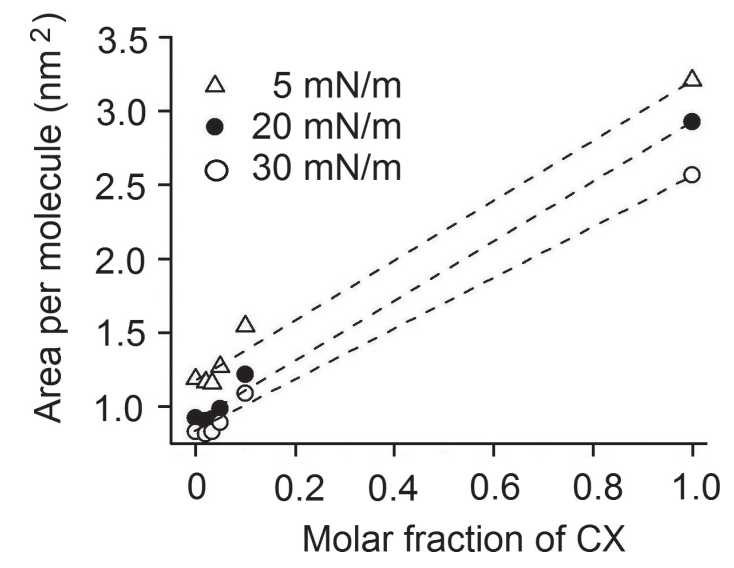

Figure 2. The plot of mean molecular area $v s$. molar fraction of CX in the DPhPC monolayers calculated according to Eq. (5) for three values of surface pressures. The dashed lines represent the mean molecular area corresponding to ideal miscibility of the components.

concentration of lipids for experimentally obtained values (presented in Table 1) and for those calculated based on the data for area per molecule of pure lipid $\left(1.04 \mathrm{~nm}^{2}\right)$ and pure CX $\left(3.32 \mathrm{~nm}^{2}\right.$ ) (Fig. 3). The calculated ,ideal“ values of mean molecular area correspond to the ideal miscibility of both components. For example the "ideal" mean area per molecule for molar ratio $\mathrm{DPhPC} / \mathrm{CX}=50: 1$ (corresponding to the lipid relative concentration: 50 ) can be calculated as follows: [50 $\times$ $\left.1.04 \mathrm{~nm}^{2}+1 \times 3.32 \mathrm{~nm}^{2}\right] / 51=1.08 \mathrm{~nm}^{2}$ per molecule. As it can be seen from Fig. 3, both theoretical (ideal) as well as

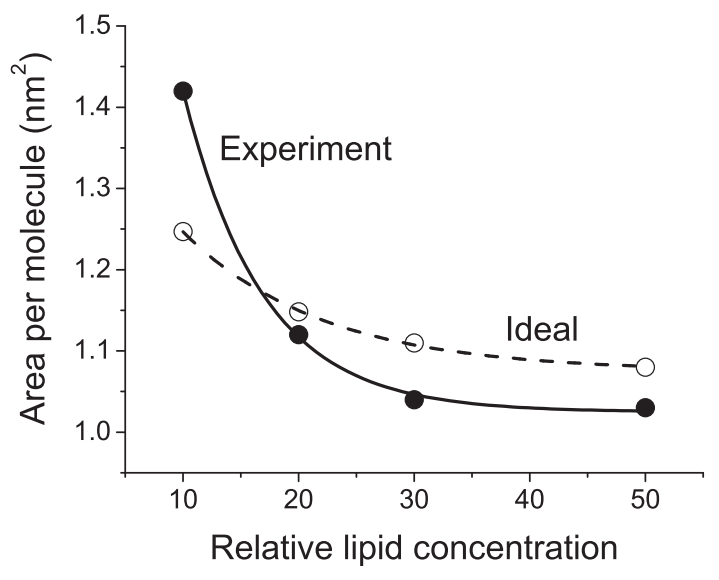

Figure 3. The plot of mean molecular area vs. relative lipid concentration in the mixed DPhPC-CX monolayers for experimentally obtained data (presented in Table 1) (curve marked as "Experiment") and for values calculated using the mean molecular area of lipids and CX (curve marked as "Ideal") corresponding to ideal miscibility. See the text for more explanation. experimentally obtained data are well fitted by exponential function. These curves crosses at the molar ratio DPhPC/CX $=17: 1$. This means that at this molar ratio the ideal miscibility takes place. At higher molar ratios the stable complexes between the lipids and CX are formed, while at lower molar ratios the CX start to form aggregates.

Hence, the obtained results suggest subtle differences in miscibility of CX in DPhPC monolayers. It is likely that additional study involving for example atomic force microscopy (AFM) or scanning electron microscopy (SEM) are required for obtaining better insight into the structure of the monolayers. We should note that the shift of the $\pi$-A isotherms toward higher molecular area with increased content of CX has been obtained also for monolayers composed of DPPC as well as for that composed of negatively charged dipalmitoylphosphatidyl glycerol (DPPG) monolayers (results are not shown).

\section{Mechanical properties of monolayers}

More detailed information about the physical properties of the monolayers can be acquired from the interfacial elastic modulus of area compressibility, $\mathrm{C}_{\mathrm{S}}{ }^{-1}$, defined as (Capuzzi et al. 2000):

$$
\mathrm{C}_{\mathrm{S}}^{-1}=-\mathrm{A}(\delta \pi / \delta \mathrm{A})
$$

The quantity $(\delta \pi / \delta \mathrm{A})$ is the slope of the monolayer and the area, $\mathrm{A}$, corresponds to the mean molecular area at the indicated surface pressure, $\pi$. According to Capuzzi et al. (2000), the values of modulus of area compressibility between 0 to $12.5 \mathrm{mN} / \mathrm{m}$ refer to the gas phase of the films, from 12.5 to $50 \mathrm{mN} / \mathrm{m}$ to the liquid - expanded (LE) films, from 100 to $250 \mathrm{mN} / \mathrm{m}$ to the liquid - condensed (LC) films and the values above $250 \mathrm{mN} / \mathrm{m}$ are characteristic for the solid films (S). Using Eq. (6), we determined the compressibility modulus for condensed state of the monolayers. Analogically to the model reported in Sabatini et al. (2008) we constructed plots of the area compressibility modulus as a function of area per molecule and surface pressure for monolayers composed of DPhPC/CX (Fig. 4 A,B). The above mentioned dependences for monolayers of pure phospholipids and for mixed phospholipid-calixarene monolayers did not differ significantly except the DPhPC/CX molar ratio 10:1 for which the maximum of the compressibility modulus was shifted toward higher molecular areas. This result agrees well with the weak effect of the calixarenes on the shape of $\pi-A$ isotherms (except of the increase of the area per molecule and increase of the critical pressure with increasing the molar fraction of calixarenes) reported above. As it can be seen from Fig. 4, the area compressibility moduli start to increase monotonously as soon as the surface increases. The decrease of the compressibility modulus prior 
A

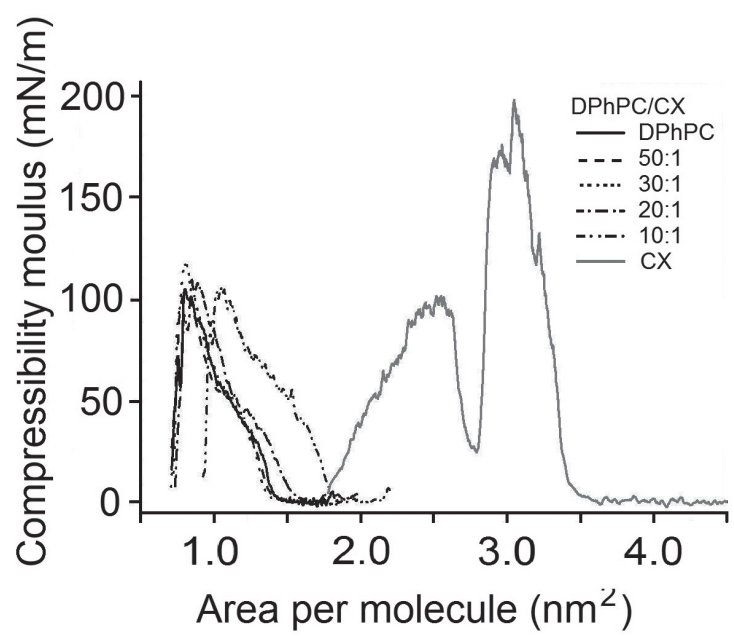

B

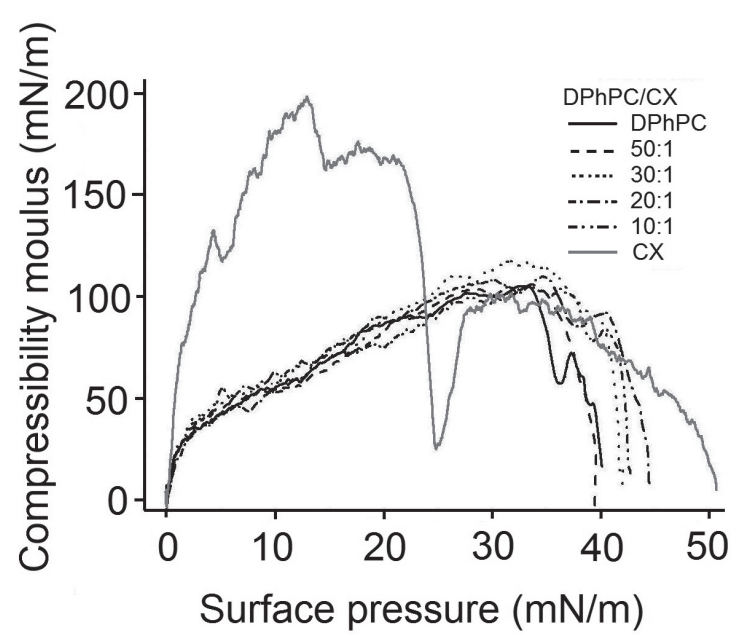

Figure 4. The plot of the area compressibility modulus vs. molecular area (A) and surface pressure (B) for DPhPC monolayers containing CX in different molar ratios (see the legend).

to critical pressure is probably connected with the fact that the collapse of the monolayer does not exactly start at the moment of critical pressure but earlier. Situation illustrated on Fig. 4B indicates that the monolayer degradation starting $\sim 5-8 \mathrm{mN} / \mathrm{m}$ below the critical pressure. The possible reason of this effect, as well as the drop of the surface potential just before the collapse, could be due to continuous breakage of the monolayer once it undergoes the transition into the more compact solid phase. In this phase the first rupture in the monolayer might be due to appearance of some structural defects. Under the further compressing, the size and the area of the defects increases and monolayer is slowly losing its two-dimensional structure. We suppose that the real collapse of the monolayer takes place not at the collapse pressure but in the solid state of the monolayer where the compressibility and surface potentials possess the highest values. Thus, exactly when the elasticity modulus started to decrease the first inhomogenities appear and the monolayer lost the integrity. Further studies of this phenomenon are, however, required based in particularly on the analysis of the possible hysteresis of the $\pi$ - $A$ isotherms at compressiondecompression process.

Sharp decrease of area compressibility modulus of the monolayers composed of pure CX at molecular area 2.75 $\mathrm{nm}^{2}$ and at surface pressure $25 \mathrm{mN} / \mathrm{m}$ is related to the existence of the region of constant surface pressure, i.e. plateau at the $\pi$-A isotherm (see Fig. $1 \mathrm{~A}$ ). In this region the structural changes of the monolayer of pure CX took place. The values of compressibility modulus before and after the phase transition are in the interval $100-200 \mathrm{mN} / \mathrm{m}$. This means that monolayer changes its structure in framework of LC phase (Pekalski 1988).
The maximal values of compressibility modulus for monolayers studied are shown in Table 1. It can be seen that the compressibility moduli of pure DPhPC monolayers and their mixtures with CX are between 101-117 mN/m, which suggest that at maximal compression close to the critical pressure these monolayers are in LC phase. However, the compressibility modulus of pure CX monolayers is much higher $(195.3 \pm 2.1 \mathrm{mN} / \mathrm{m})$.

\section{The effect of cyt $c$ on the surface pressure of monolayers composed of CX at water subphase}

As we mentioned above, CX specifically binds cyt c. The specific binding is probably due to cooperative recognition between the negatively charged $-\mathrm{COO}^{-}$groups on the outer rim of CX rather than of the unspecific electrostatic interactions in analogy with tetraanionic calixarene tetraphosphonate 1 reported by Zadmard et al. (2004). In our recent work we studied effect of this protein on the molecular area of CX formed at water subphase containing various concentration of cyt c (Hianik et al. 2011). We have shown that with increased concentration of cyt $c$ the $\pi$-A isotherms shift toward higher area per molecule. This may be due to the binding of cyt $\mathrm{c}$ to $\mathrm{CX}$, which forms bulkier complexes with limited ability of each complex to approach each other and possible incorporation of cyt $\mathrm{c}$ into the monolayer. The plot of the relative changes of the molecular area $v s$. concentration of cyt c had the shape of Langmuir isotherm. By fitting the results using Langmuir equation we determined the equilibrium constant of dissociation, $\mathrm{K}_{\mathrm{D}}=26.0 \pm 5.4 \mathrm{nM}$, which suggests strong binding of cyt $\mathrm{c}$ to the $\mathrm{CX}$. The interaction of cyt c with CX may, however, depend on the phase state of 


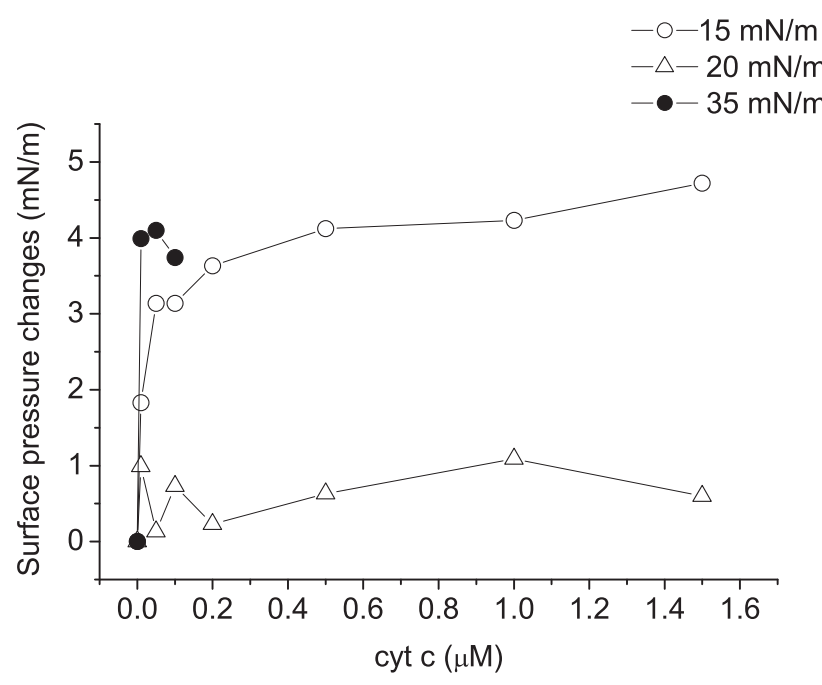

Figure 5. The plot of the changes of surface pressure $v s$. concentration of cyt $\mathrm{c}$ in a water subphase for different initial surface pressure of the monolayer formed by CX.

the CX monolayer. Therefore, in this work we studied the interaction of cyt $\mathrm{c}$ with $\mathrm{CX}$ monolayers at various surface pressures: $5,15,20$ and $35 \mathrm{mN} / \mathrm{m}$, i.e. when the monolayer is in liquid-expanded ( 5 and $15 \mathrm{mN} / \mathrm{m}$ ), liquid condensed $(20 \mathrm{mN} / \mathrm{m})$ or solid $(35 \mathrm{mN} / \mathrm{m})$ state, respectively. In these experiments the CX monolayer was formed at the PBS subphase in a Teflon cell of the volume $7 \mathrm{ml}$. After the surface pressure was stabilized, the cyt $\mathrm{c}$ was titrated directly into the subphase via the small hole in Teflon in order to avoid disturbance of the monolayer. The plot of the changes of surface pressure $v s$. cyt c concentration is shown on Fig. 5. As it can be seen from this figure, for relatively low surface pressure $(15 \mathrm{mN} / \mathrm{m})$ when the monolayer is in LE state, the addition of low concentration of cyt $c$ resulted in sharp increase of the surface pressure (see also Fig. 6, curve 1 for surface pressure $5 \mathrm{mN} / \mathrm{m}$ ). Much smaller changes of the surface pressure were observed for monolayers in LC state. Completely different behavior of the surface pressure changes took place for monolayers in solid state. In this case the monolayer is rather compact. After initial rise of the surface pressure following addition of cyt $\mathrm{c}$ at concentration $10 \mathrm{nM}$, the surface pressure became unchanged after subsequent cyt $\mathrm{c}$ additions. We assume that at this low concentration, cyt $\mathrm{c}$ binds to the most of CX binding sites. Therefore, further increase of cyt $c$ in a subphase has only minor effect on the surface pressure. Thus, most pronounced changes in a surface pressure following addition of cyt $\mathrm{c}$ were observed for the monolayer in LE state. At this state the monolayer is not well ordered, the molecules of CX have relatively large rotational freedom and consequently also various orientations. Therefore, the probability of capturing cyt c molecules is rather large in

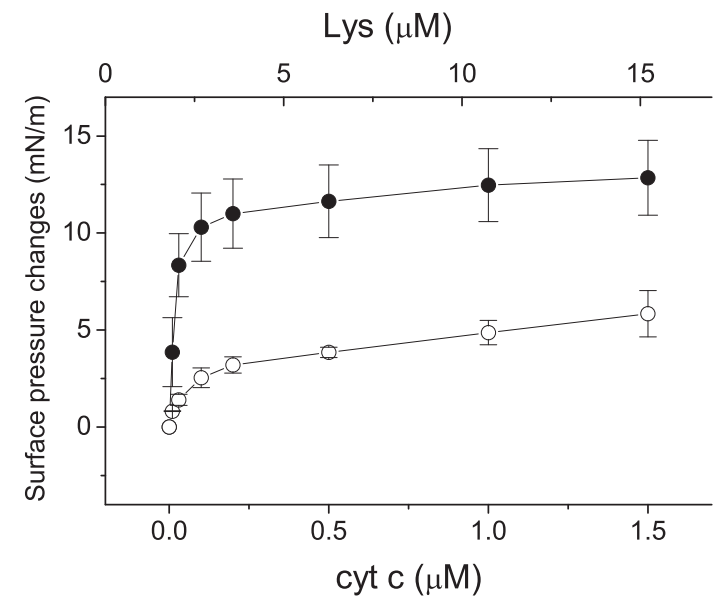

Figure 6. The plot of the changes of surface pressure $v s$. concentration of cyt c $(\bullet)$ and lysine $(O)$ in a water subphase for the monolayer composed of CX. The value of initial surface pressure was $5 \mathrm{mN} / \mathrm{m}$. The results represent mean $\pm \mathrm{SD}$ obtained from 3 experiments in each series.

comparison with tightly packed CX monolayers at higher surface pressures. Another reason of the surface pressure increase at LE state of the monolayer could be penetration of cyt $c$ into the air-water interface. Such effect has been already observed for lipid monolayers at lower surface pressures $(\pi<$ $20 \mathrm{mN} / \mathrm{m}$ ) (Zhao et al. 2002; Gorbenko et al. 2009). Cyt $\mathrm{c}$ is larger in comparison with CX; however, after the adsorption at the air-water interface it may not necessarily interact with negatively charged carboxylic groups of CX. Therefore, cyt $\mathrm{c}$ and CX could coexist in the monolayer even without substantial interaction. For higher surface pressures there is not sufficient space at the surface for cyt c penetration. Therefore, the electrostatic interactions between cyt $c$ and CX are dominant. Binding of cyt $\mathrm{c}$ to the CX resulted in formation of the complex with the lower movement degree freedom of CX molecules. At the same time such a complex is more robust. Because CX molecules can not be more close to each other, any subsequent addition of cyt $\mathrm{c}$ should result in increase of surface pressure and possibly of the collapse of the monolayer.

We should mention that in this work we have focused on the study of interaction of cyt $\mathrm{c}$ with monolayers formed from CX only, and not with mixed DPhPC-CX structures. This is in particularly due to the fact that cyt $\mathrm{c}$ interacts with lipid monolayers of DPhPC (results are not shown), mostly because this phosholipid is in wide temperature range $\left(-120\right.$ to $\left.+120^{\circ} \mathrm{C}\right)$ in a liquid crystalline state (Lindsey et al. 1979). Therefore the interaction of cyt $c$ with such mixed monolayers should be more complex in comparison with pure CX monolayers. In particularly, it can be expected that in a mixed monolayers the apparent $\mathrm{K}_{\mathrm{D}}$ value could 
be higher indicating decrease of the affinity of cyt $c$ to the receptors. Certainly, using impedance spectroscopy that has been applied to a mixed supported films composed of soy bean phosphatidylcholine (SBPC) and CX in molar ratio $\mathrm{SBPC} / \mathrm{CX}=10: 1$, the $\mathrm{K}_{\mathrm{D}}$ value was $66.1 \pm 21.2 \mathrm{nM}$, which is more than twice higher in comparison with the $K_{D}$ value typical for CX monolayers. (SBPC film was also in a liquid crystalline state) (Garaiova et al. 2012). At the same time, the interaction of cyt $\mathrm{c}$ with CX monolayers was specific. This has been shown in the experiments where instead of cyt $c$, lysine (Lys) was used. Lys also incorporates into the CX cavity due to its amino group. However, the comparable increase of the surface pressure has been achieved at more than 10 times higher concentration of Lys in a water subphase in comparison with cyt c (see Fig. 6).

\section{Viscoelastic properties of BLM contained CX}

The area compressibility modulus of lipid monolayers provides information on the mechanical properties of lipid films in lateral direction. For BLM we used special electrostriction method that allows us to analyze compressibility and dynamic viscosity of lipid bilayer in direction perpendicular to the membrane plane. The Young modulus of elasticity, $\mathrm{E}_{\perp}$, which characterizes the mechanical properties of BLM is sensitive to the structural state of the hydrophobic part of the bilayers (Hianik 2008). We also determined the coefficient of dynamic viscosity, $\eta$, which characterizes friction of the inner part of the membrane during periodic deformation. Along with viscoelastic parameters we determined also electrical capacitance, C, of the BLM. Application of $\mathrm{AC}$ voltage to the BLM resulted in time-dependent changes of above-mentioned parameters. However after certain decrease at the beginning of the kinetics due to formation of BLM these parameters reached steady-state values (results are not shown). The average values of $E_{\perp}, \eta$ and $C$ for BLM composed of DPhPC and CX at two molar ratios (DPhPC/ $\mathrm{CX}=100: 1$ and 50:1) are shown on Fig. 7. The BLM was stable only until relatively low concentration of CX (molar ratio 50:1). At higher concentration of CX BLMs tended to break down abruptly few second after membrane formation took place, or it was impossible to form bilayers at all. The reason of BLM instability may be connected with formation of aggregates and as a result in appearance the structural defects at the borders between the calixarenes and the phospholipid bilayer. Elasticity modulus and coefficient of dynamic viscosity of BLMs containing CX were higher in comparison with unmodified BLM. This suggests that CX causes ordering of the lipid bilayer.

Thus the physical properties of lipid monolayers and BLM composed of phospholipids and calixarenes gave us opportunity to obtain important information on the stability of the self-assembled layers and on possible complex formation

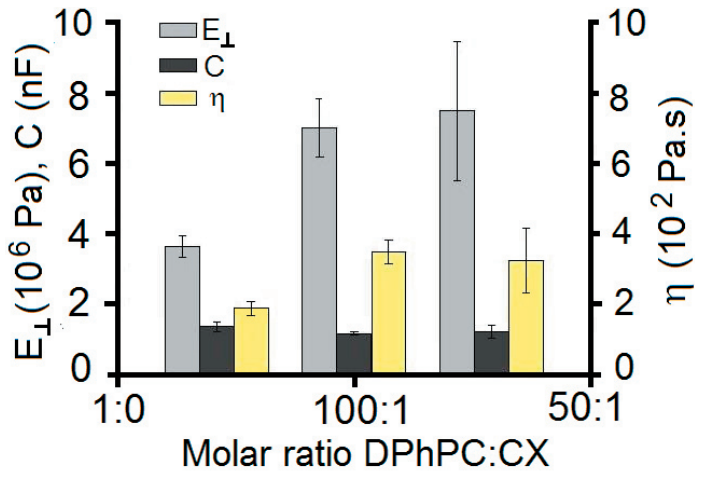

Figure 7. The diagram of the steady-state values of electrical capacitance, $\mathrm{C}$, elasticity modulus, $\mathrm{E}_{\perp}$, and coefficient of dynamic viscosity, $\eta$, of BLM composed of DPhPC and cholesterol (4:1 w/w) and various molar ratios of $\mathrm{CX}$. The results represent mean $\pm \mathrm{SD}$ obtained from 6 BLMs in each series.

between $\mathrm{CX}$ and phospholipids. The area compressibility modulus, $\mathrm{C}_{\mathrm{S}}{ }^{-1}$, as well as the Young modulus of elasticity, $\mathrm{E}_{\perp}$, increase with increasing calixarene concentration. This suggests ordering effect of calixarenes on the monolayers and on the BLM hydrophobic part. Cyt $\mathrm{c}$ in a water subphase interacts with $\mathrm{CX}$ in monolayers depending on their phase state. The most pronounced increase of surface pressure of monolayers following addition of cyt $\mathrm{c}$ into the water subphase was observed for monolayers in relatively low ordered liquid expanded state (LE). This can be due to larger molecular freedom in CX orientation as well as possibility of penetration of cyt $c$ into the air-water interface.

\section{Conclusion}

In this work we studied the thermodynamical and mechanical properties of lipid monolayers and BLM composed of $\mathrm{DPhPC}$ and contained various concentration of calixarene that specifically bind cyt $\mathrm{c}$. The studies on monolayers showed, that with increased content of CX the $\pi$-A isotherms shift toward higher molecular area. The analysis on the monolayer component miscibility indicates that at relatively low molar ratios of $\mathrm{DPhPC} / \mathrm{CX}=17: 1$ the ideal miscibility takes place, while at higher molar ratios the complexes between the phospholipids and CX are formed. Higher content of CX, however, resulted in appearance of calixarene aggregates. The analysis of mechanical properties of the DPhPC monolayers suggests that they are rather soft in comparison with pure CX monolayers. The area compressibility modulus of DPhPC was $104.5 \pm 2.0 \mathrm{mN} / \mathrm{m}$, which is much smaller in comparison with that of pure CX monolayer $(195.3 \pm 2.1 \mathrm{mN} / \mathrm{m})$. Interestingly, the compressibility modulus of DPPC monolayer in a gel state is rather high $(197.8 \pm 5.5 \mathrm{mN} / \mathrm{m})$ (Vitovic et al. 
2006) and comparable with that of rigid CX monolayer. This suggests that the branched hydrocarbon chains of DPhPC provide substantial fluidity of the monolayer.

The interesting peculiarity of this study is observation of the increase of the surface pressure of the lipid monolayers composed of CX following addition of cyt c. This increase was much sharper for relatively low surface pressure (5-15 $\mathrm{mN} / \mathrm{m}$ ) and can be connected with complicated behaviour of cyt $\mathrm{c}$ at the air-water interface where the protein can interact with CX or even adsorb at the interface between CX molecules. In contrast, much weaker effect on surface pressure changes has been observed when lysine instead of cyt $\mathrm{c}$ was added into the water subphase.

The study of mechanical properties of BLM contained CX confirmed increase of elasticity modulus in direction perpendicular to the membrane plane, $\mathrm{E}_{\perp}$, with increasing $\mathrm{CX}$ concentration. In this respect the results confirm the effect of CX on the rigidity of the lipid film. However, in contrast with monolayers where the compressibility modulus reflects the molecular interactions in lateral direction, the increase in $E_{\perp}$ value of BLM reflects also increased ordering of the BLM hydrophobic part induced by CX.

Acknowledgements. This work was supported by Agency for Promotion Research and Development under the contracts No. APVV-041010, LPP-0341-09, LPP-0250-09 and VEGA grant agency (project No. $1 / 0785 / 12$ ). This publication is also the result of the project implementation: BIOMAKRO2, ITMS: 26240120027, supported by the Research \& Development Operational Programme funded by the ERDF. We thank to Dr. T. Oshima for generous gift of calixarenes.

\section{References}

Baldini L., Casnati A., Sansone F., Ungaro R. (2007): Calixarenebased multivalent ligands. Chem. Soc. Rev. 36, 254-266 http://dx.doi.org/10.1039/b603082n

Barcena-Uribarri I., Thein M., Sacher A., Bunikis I., Monde M., Bergström S., Benz R. (2010): P66 porins are present in both Lyme disease and relapsing fever spirochetes: A comparison of the bipophysical properties of $\mathrm{P} 66$ porins from six Borellia species. Biochim. Biophys. Acta 1798, 1197-1203 http://dx.doi.org/10.1016/j.bbamem.2010.02.011

Brockman H. (1994): Dipole potential of lipid membranes. Chem. Phys. Lipids 73, 57-79 http://dx.doi.org/10.1016/0009-3084(94)90174-0

Cacciapaglia R., Casnati, A., Mandolini L., Reinhoudt D. N., Salvio R., Sartori A., Ungaro R. (2006): Catalysis of diribonucleoside monophosphate cleavage by water soluble copper (II) complexes of calyx[4]arene based nitrogen ligands. J. Am. Chem. Soc. 128, 12322-12330 http://dx.doi.org/10.1021/ja0632106

Capuzzi G., Fratini E., Pini F., Baglioni P., Casnati A., Teixeira J. (2000): Counterion complexation by calixarene ligands in cesium and potassium dodecyl micelles. A small angle neutron scattering study. Langmuir 16, 188-194 http://dx.doi.org/10.1021/la9907580

Cseh R., Benz R. (1998): The adsorption of phloretin to lipid monolayers and bilayers cannot be explained by Langmuir adsorption isotherms alone. Biophys. J. 74, 1399-1408 http://dx.doi.org/10.1016/S0006-3495(98)77852-X

Dei L., Casnati A., LoNostro P., Baglioni P. (1995): Selective complexation by p-tert-butylcalix[6]arene in monolayers at the water-air interface. Langmuir 11, 1268 - 1272 http://dx.doi.org/10.1021/la00004a037

Deshmukh V., Britt D. W., Hlady V. (2010): Excess fibrinogen adsorption to monolayers of mixed lipids. Coll. Surf. B. 81, 607-613 http://dx.doi.org/10.1016/j.colsurfb.2010.08.008

Discher B. M., Schief W. R., Vogel V., Hall S. B. (1999): Phase separation in monolayers of pulmonary surfactant phospholipids at the air-water interface: composition and structure. Biophys. J. 77, 2051-2061 http://dx.doi.org/10.1016/S0006-3495(99)77046-3

Garaiova Z., Mohsin M. A., Vargova V., Banica F-G., Hianik T. (2012): Electrochemical impedance spectroscopy for assessing the recognition of cytochrome $\mathrm{c}$ by immobilized calixarenes. Bioelectrochemistry 87, 220-225

Gorbenko G. P. Trusova V. M., Molotkovsky J. G., Kinnunen P. K. J. (2009): Cytochrome c induces lipid demixing in weakly charged phosphatidylcholine/phosphatidylglycerol model membranes as evinced by resonance energy transfer. Biochim. Biophys. Acta 1788, 1358-1365

Guntupalli R., Sorokulova I., Long R., Olsen E., Neely W., Vodyanoy V. (2011): Phage Langmuir monolayers and Langmuir-Blodgett films. Coll. Surf. B. 82, 182-189 http://dx.doi.org/10.1016/j.colsurfb.2010.08.032

Guo X., Lu G. Y., Li Y. (2004): Interaction between calyx[4]arene derivative bearing adenino units and complementary nucleosides at the air-water interface. Thin Solid Films 460, 264-268 http://dx.doi.org/10.1016/j.tsf.2004.01.108

Hassen W. M., Martelet C., Davis F., Higson S. P. J., Abdelghani A., Helali S., Jaffrezic-Renault N. (2007): Calix[4] arene based molecules for amino-acid detection. Sensors and Actuators B 124, 38-45 http://dx.doi.org/10.1016/j.snb.2006.11.044

Hianik T., Passechnik V. I. (1995): Bilayer Lipid Membranes: Structure and Mechanical Properties. Kluwer Academic Publishers, Dordrecht

Hianik T. (2008): Biological membranes and membrane mimics. In: Bioelectrochemistry. Fundamentals, Experimental Techniques and Applications. (Ed. P. N. Bartlett), pp. 87-156, Wiley, Chichester

Hianik T., Garaiova Z., Vargova V., Vitovic P. (2011): The study of the interaction of cytochrome $\mathrm{c}$ with calixarenes incorporated into the lipid membranes. Czechoslovak J. Physics 61, 194-198

Houel E., Lazar A., Da Silva E., Coleman A. W., Solovyov A., Cherenok S., Kalchenko V. I. (2002): Interfacial interactions of cations with amphiphilic dihydroxyphosphonyl-calix[4]arene mesosystems. Langmuir 18, 1374-1379 http://dx.doi.org/10.1021/la011242v

Hsieh C. H., Sue S. C., Lyu P. C., Wu W. G. (1997): Membane packing geometry of diphytanoylphosphatidylcholine is highly sensitive to hydration phospholipid polymorphism induced by 
molecular rearrangement in the headgroup region. Biophys. J. 73, 870-877 http://dx.doi.org/10.1016/S0006-3495(97)78120-7

Izzo I., Licen S., Maulucci N., Autore G., Marzocco S., Tecilla P., De Riccardis F. (2008): Cationic calix[4]arenes as anion-selective ionophores. Chem. Commun. 14, 2986-2988 http://dx.doi.org/10.1039/b719482j

Jin T. (2007): Calixarene-based photoresponsive carrier for the control of $\mathrm{Na}+$ flux across a lipid bilayer membrane by visible light. Mater. Lett. 61, 805-808 http://dx.doi.org/10.1016/j.matlet.2006.05.064

Kates M., Yengoyan L. S., Sastry P. S. (1965): A diether analog of phosphatidyl glycerophosphate in halobacterium cutirubrum. Biochim. Biophys. Acta 98, 252-268 http://dx.doi.org/10.1016/0005-2760(65)90119-0

Li C., Huang J., Liang Y. (2001): Structure control on photodimerization of uracil and thymine moieties in nucleolipid Langmuir-Blodgett films by the molecular recognition effect at the air/water interface. Langmuir 17, 2228-2234 http://dx.doi.org/10.1021/la001105i

Lindsey H., Petersen N. O., Chan S. I. (1979): Physicochemical characterization of 1,2-diphytanoyl-sn-glycero-3-phosphocholine in model membrane systems. Biochim. Biophys. Acta $\mathbf{5 5 5}, 147-167$ http://dx.doi.org/10.1016/0005-2736(79)90079-8

Liu F., Lu G. Y., He W. J., Liu M. H., Zhu L. G. (2002): Supramolecular interaction of diamino calix[4] arene derivative with nucleotides at the air-water interface. Thin Solid Films 414, 72-77 http://dx.doi.org/10.1016/S0040-6090(02)00433-9

Lonetti B., Fratini E., Casnati A., Baglioni P. (2004): Langmuir monolayers of calix[8]arene derivates: complexation of alkaline earth ions at the water-air interface. Coll. Surfaces A 248, $135-143$ http://dx.doi.org/10.1016/j.colsurfa.2004.06.036

Ludwig R. (2000): Calixarenes in analytical and separation chemistry. Fresn. J. Anal. Chem. 367, 103-128 http://dx.doi.org/10.1007/s002160051611

Ludwig R., Dzung T. K. (2002): Calixarene-based molecules for cation recognition. Sensors 2, 397-416 http://dx.doi.org/10.3390/s21000397

Ludwing R. (2005): Calixarenes for biochemical recognition and separation. Microchim. Acta 152, 1-19 http://dx.doi.org/10.1007/s00604-005-0422-8

Maget-Dana R. (1999): The monolayer technique: a potential tool for studying the interfacial properties of antimicrobial and membrane-lytic peptides and their interactions with lipid membranes. Biochim. Biophys. Acta 1462, 109-140 http://dx.doi.org/10.1016/S0005-2736(99)00203-5

Mirsky V. M., Mass M., Krause C., Wolfbeis O. S. (1998): Capacitive approach to determine phospholipase A2 activity toward artificial and natural substrates. Anal. Chem. 70, 3674-3678 http://dx.doi.org/10.1021/ac980102w

Mohsin M. A., Banica F-G., Oshima T., Hianik T. (2011): Electrochemical impedance spectroscopy for assessing the recognition of cytochrome c by immobilized calixarenes. Electroanalysis 23, 1229-1235 http://dx.doi.org/10.1002/elan.201000686
Mueller P., Rudin D. O., Tien H. T., Wescott W. C. (1962): Reconstitution of cell membrane structure in vitro and its transformation into an excitable system. Nature 194, 979-980 http://dx.doi.org/10.1038/194979a0

Nikolelis D. P., Theoharis G. (2003): Biosensor for dopamine based on stabilized lipid films with incorporated resorcin[4]arene receptor. Bioelectrochemistry 59, 107-112 http://dx.doi.org/10.1016/S1567-5394(03)00009-4

Nikolelis D. P., Hianik T., Nikoleli G-P. (2010): Stabilized lipid films in electrochemical biosensors. Electroanalysis 22, 2747-2763 http://dx.doi.org/10.1002/elan.201000420

Oh S. W., Moon J. D., Lim H. J., Park S. Y., Kim T., Park J. B., Han M. H., Snyder M., Choi E. Y. (2005): Calixarene derivative as a tool for highly sensitive detection and oriented immobilization of proteins in a microarray format through noncovalent molecular interaction. FASEB 19, 1335-1337

Omar O., Ray A. K., Hasan A. K., Davis F. (1997): Resorcin calixarenes (resorcinarenes): Langmuir-Blodgett films and optical properties. Supramol. Sci. 4, 417-421 http://dx.doi.org/10.1016/S0968-5677(97)00024-2

Oshima T., Higuchi H., Ohto K., Inoue K., Goto M. (2005): Selective extraction and recovery of cytochrome $\mathrm{c}$ by liquid-liquid extraction using a calix[6]arene carboxylic acid derivative. Langmuir 21, 7280-7284 http://dx.doi.org/10.1021/la050364a

Oshima T., Suetsugu A., Baba Y. (2010): Extraction and separation of lysine-rich protein by formation of supramolecule between crown ether and protein in aqueous two-phase system. Anal. Chim. Acta 674, 211-219 http://dx.doi.org/10.1016/j.aca.2010.06.039

Pekalski A. (1988): Phase diagrams for a model of a lipid monolayer. Eur. Biophys. J. 16, 39-44 http://dx.doi.org/10.1007/BF00255324

Petty M. C. (1996): Langmuir-Blodgett Films. An Introduction, Cambridge University Press, Cambridge http://dx.doi.org/10.1017/CBO9780511622519

Sabatini K., Mattila J. P., Kinnunen P. K. J. (2008): Interfacial Behavior of Cholesterol, Ergosterol, and Lanosterol in Mixtures with DPPC and DMPC. Biophys. J. 95, 2340-2355 http://dx.doi.org/10.1529/biophysj.108.132076

Schug Z. T., Gottlieb E. (2009): Cardiolipin acts as a mitochondrial signalling platform to launch apoptosis. Biochim. Biophys. Acta 1788, 2022-2031 http://dx.doi.org/10.1016/j.bbamem.2009.05.004

Shapovalov V. I. (1998): Effect of gramicidin A on the dipole potential of phospholipid membranes. Thin Solid Films 327-329, 299-305

Sidorov V., Kotch F. W., Abdrakhmanova G., Mizani R., Fettinger J. C., Davis J. T. (2002): Ion channel formation from a calix[4]arene amide that binds HCl. J. Am. Chem. Soc. 124, 2267-2278 http://dx.doi.org/10.1021/ja012338e

Sliwa W., Deska M. (2011): Functionalization reactions of calixarenes. ARKIVOC 496-551 http://dx.doi.org/10.3998/ark.5550190.0012.110

Slottrup B. J., Stevens D. S., Keller S. I. (2005): Miscibility of ternary mixtures of phospholipids and cholesterol in monolayers, and application to bilayer systems. Biophys. J. 88, 269-276 http://dx.doi.org/10.1529/biophysj.104.048439 
Sugden M. W., Richardson T. H., Davis F., Higson S. P. J., Faul C. F. J. (2008): Langmuir and LB properties of two calyx[4]resorcinarenes: Interactions with various analytes. Coll. Surf. A 321, 43-46 http://dx.doi.org/10.1016/j.colsurfa.2008.02.048

Teuntje M., Andriessen J. C., Jacobs B., Vos P. E. (2010): Clinical characteristics and patophysiological mechanisms of focal and diffuse traumatic brain injury. J. Cell. Mol. Med. 14, 2381-2392 http://dx.doi.org/10.1111/j.1582-4934.2010.01164.x

Turshatov A. A., Melnikova N. B., Semchikov Yu. D., Ryzhkina I. S., Pashirova T. N., Möbius D., Zaitsev S. Yu. (2004): Interaction of monolayers of calix[4] resorcinarenes derivatives with copper ions in aqueous subphase. Coll. Surf. A. Phys. Eng. Asp. 240, 101-106 http://dx.doi.org/10.1016/j.colsurfa.2004.02.031

van der Heyden A., Regnouf-de-Vains J. B., Warszynski P., Dalbavie J. O., Zywoczynski A., Rogalska E. (2002): Probing inter- and intramolecular interactions of six new p-tert-butylcalix[4]arenebased bipyridil podans with Langmuir monolayers. Langmuir 18, $8854-8861$ http://dx.doi.org/10.1021/la025575j

Vitovic P., Nikolelis D. P., Hianik T. (2006): Study of calix[4]resorcinarene-dopamine complexation in mixed phospholipid monolayers formed at the air-water interface. Biochim. Biophys. Acta 1758, 1852-1861 http://dx.doi.org/10.1016/j.bbamem.2006.08.011
Vollhardt D., Gloede J., Weidemann G., Rudert R. (2003): Characteristic features of amphiphilic p-functionalized calixarene monolayers at the air/water interface. Langmuir 19, $4228-4234$ http://dx.doi.org/10.1021/la026856u

Weis M., Janicek R., Cirak J., Hianik T. (2007): Study of the calix[4]resorcinarene-dopamine interactions in monolayers by measurement of pressure-area isotherms and Maxwell displacement currents. J. Phys. Chem. B. 111, 10626-10631 http://dx.doi.org/10.1021/jp0741051

Ye Z., Pang S., He W., Shi X., Guo Z., Zhu L. (2001): Copper (III) ion induced monolayers formation of p-tert-butylthiacalix[4] arene at the air-water interface. Spectrochim. Acta A 57, 14431447 http://dx.doi.org/10.1016/S1386-1425(00)00485-6

Zadmard R., Arendt M., Schrader T. (2004): Multipoint recognition of basic proteins at a membrane model. J. Am. Chem. Soc. 126, 7752-7753 http://dx.doi.org/10.1021/ja049191m

Zhao H., Dubielecka P. M., Söderlund T., Kinnunen P. K. J. (2002): Interactions of adriamycin, cytochrome $\mathrm{c}$, and serum albumin with lipid monolayers containing poly(ethylene glycol)-ceramide. Biophys. J. 83, 954-967

http://dx.doi.org/10.1016/S0006-3495(02)75221-1

Received: September 19, 2012

Final version accepted: January 8, 2013 